\title{
Pulmonary lesions associated with sputum culture-positive actinomycetes: report of one case
}

\author{
Lingwei Wang ${ }^{1 \#}$, Heng Zhang ${ }^{1 \#}$, Di Wu ${ }^{1 \#}$, Mengjie Feng ${ }^{1}$, Peng Yang ${ }^{1}$, Xiaoyi Hu ${ }^{1}$, Pierre Tattevin ${ }^{2}$, \\ Goohyeon Hong ${ }^{3}$, Rongchang Chen ${ }^{1}$, Chen Qiu ${ }^{1}$
}

${ }^{1}$ Department of Respiratory and Critical Care Medicine, Shenzhen People's Hospital, Shenzhen Institute of Respiratory Diseases, Shenzhen Key Laboratory of Respiratory Diseases, Shenzhen Key Laboratory of Pathogenic Microorganisms and Bacterial Resistance, First Affiliated Hospital of Southern Science and Technology University, Second Clinical Medical College of Jinan University, Shenzhen 518020, China; ${ }^{2}$ Infectious Diseases and ICU, Pontchaillou University Hospital, 35033 Rennes cedex, France; ${ }^{3}$ Division of Pulmonary and Critical Care Medicine, Department of Internal Medicine, Dankook University Hospital, Dankook University College of Medicine, Cheonan, Republic of Korea

\#These authors contributed equally to this work.

Correspondence to: Chen Qiu; Rongchang Chen. Department of Respiratory and Critical Care Medicine, Shenzhen People's Hospital, Shenzhen Institute of Respiratory Diseases, Shenzhen Key Laboratory of Respiratory Diseases, Shenzhen Key Laboratory of Pathogenic Microorganisms and Bacterial Resistance, First Affiliated Hospital of Southern Science and Technology University, Second Clinical Medical College of Jinan University, Shenzhen 518020, China. Email: szchester@163.com; chenrc@vip.163.com.

\begin{abstract}
Pulmonary actinomycosis (PA) is a rare subacute or chronic infectious disease. As simple culture of Actinomyces in BAL, as with sputum, may represent colonization, the diagnosis of PA relies on pathological examination. The preferred treatment is long-term, high-dose penicillin. A 6-12-month-course of antibacterial treatment is the rule in extended PA, although the optimal duration of treatment has not been investigated through randomized trial. In this article, we report a case presented with slowly-progressing pulmonary cavitary lesions. Actinomyces odontolyticus was detected in sputum specimen harvested by tracheoscopy. The clinical diagnosis was PA, which gradually improved with prolonged treatment of penicillin and ornidazole. This is followed by a discussion of diagnosis and treatment, especially in terms of treatment.
\end{abstract}

Keywords: Pulmonary actinomycosis (PA); anaerobic bacteria

Submitted Oct 28, 2019. Accepted for publication Nov 05, 2019.

doi: 10.21037/atm.2019.12.38

View this article at: http://dx.doi.org/10.21037/atm.2019.12.38

\section{Introduction}

Pulmonary actinomycosis (PA) is a rare subacute or chronic infectious disease. Due to its atypical clinical and imaging features and low culture-positive rate, $\mathrm{PA}$ is often misdiagnosed as a tumor or tuberculosis. The diagnosis of PA relies on pathological examination, and the presence of actinomycetes hyphae and sulfur granules on biopsy can confirm the diagnosis. The preferred treatment is longterm, high-dose penicillin. In the case reported below, the patient presented with slowly-progressing intrapulmonary cavitary lesions, and Actinomyces odontolyticus was detected in sputum specimen harvested by tracheoscopy. The clinical diagnosis was PA, which gradually improved with prolonged treatment of penicillin and ornidazole.

\section{Case presentation}

The patient was a 34-year-old man, self-employed. He was admitted to our hospital on December $21^{\text {st }}, 2018$, due to "the presence of lung lesions for more than 2 years and cough/sputum production for 1 month". The patient underwent gastric polypectomy in our center more than 2 years ago (June 2016), during which chest CT (Figure 1) showed abnormal soft-tissue density and nodules in the left upper lung, which was surrounded by stripy and poorly- 


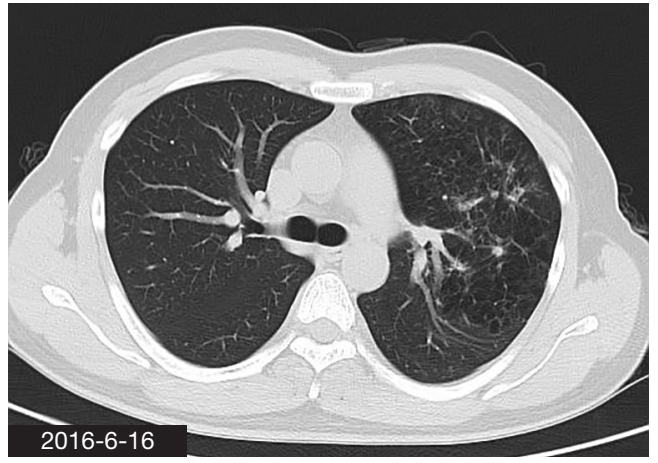

Figure 1 Irregular soft-tissue densities and nodular shadows were seen in the left upper lobe, along with stripy and poorly-defined patchy shadows in surrounding tissue. The left upper lobe was more radiolucent, along with multiple wall-free vacuoles.

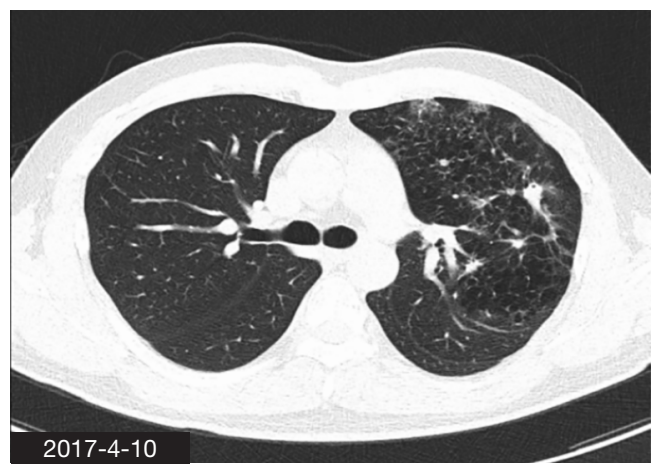

Figure 2 The number of multiple spots and nodules in the upper left lobe increased, and a consolidation shadow with a small cavity inside it was visible. The original irregular soft-tissue densities were the same as before.

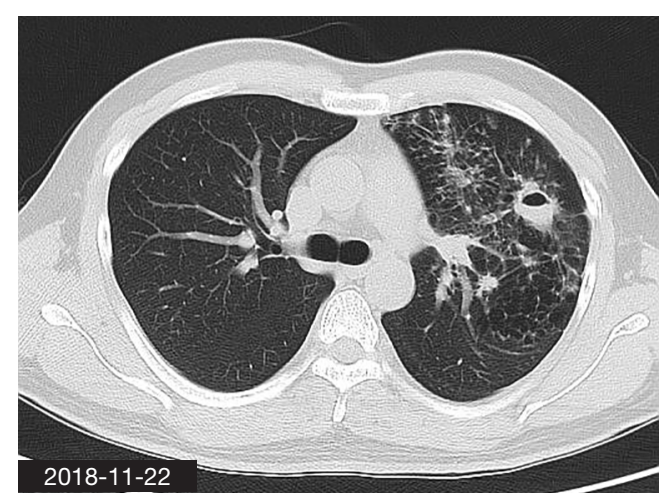

Figure 3 The cavities in left upper lobe progressed, with an increased number of exudate and stripy shadows in the surrounding tissue. defined patchy shadows. The possibility of infection, as well as left upper pulmonary emphysema and pneumatocele, were considered. Since the patient had no respiratory symptoms, no fever, and no chest pain, he received no additional investigation, and no treatment. On April $10^{\text {th }}$, 2017, a second chest CT scan was performed in another hospital (Figure 2), which revealed that the lung lesions progressed slightly. One month before his admission to our center, the patient developed cough and production of purulent sputum but without fever, chills, or nasal congestion/salivation. The symptoms improved after selfadministration of azithromycin for 3 days. On November $22^{\text {nd }}, 2018$, a lung CT scan was performed in our center (Figure 3), which revealed the consolidation of cavities in the left upper lobe, along with increased exudate from surrounding capillaries and stripy shadows. The CT scan report indicated that the lesion had further progressed; the possibility of acid-fast mycobacterial infection could not be excluded; emphysema with interstitial changes was identified in the left upper lobe, and pneumatocele in the left upper lung persisted. Bronchoscopy was performed in another hospital on December $7^{\text {th }}, 2018$, which revealed bilateral bronchial inflammation. Bronchoalveolar lavage fluid (BALF) was negative for acid-fast bacilli, and Mycobacterium tuberculosis DNA. Cough and sputum production worsened after bronchoscopy, accompanied by 2 episodes of fever (maximum body temperature, $38.4^{\circ} \mathrm{C}$ ), which improved on azithromycin. On December $18^{\text {th }}, 2018$, the patient underwent a chest CT examination in another hospital (Figure 4), which revealed the progression of the lesion in the left upper lung. He was then admitted to our hospital for diagnostic workout. Of note, the patient mental status and sleep were fair, stools and urination were normal, and there was no significant change in body weight since symptoms onset.

He had a history of repeated coughs before the age of 10 , and caries for more than 10 years, which improved after treatment. He underwent tooth extraction more than 10 years ago. In February 2017, he was operated for nasal septal deviation. He denied any history of aspiration. He had no alcohol or tobacco dependence.

Physical examination at admission showed body temperature $37^{\circ} \mathrm{C}$, heart rate $90 / \mathrm{min}$, respiratory rate 20 times $/ \mathrm{min}$, blood pressure $114 / 62 \mathrm{mmHg}(1 \mathrm{mmHg}=0.133 \mathrm{kPa})$. He had no lymphadenopathy, the pharynx was not congested, and tonsils were not swollen. Course sounds were heard in both 


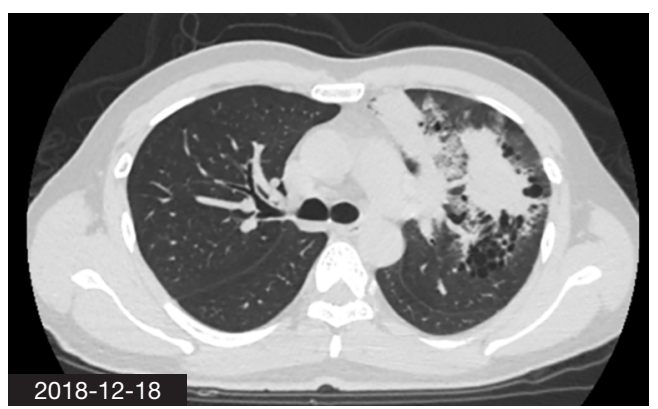

Figure 4 The exudate in the left upper lung remarkably progressed.

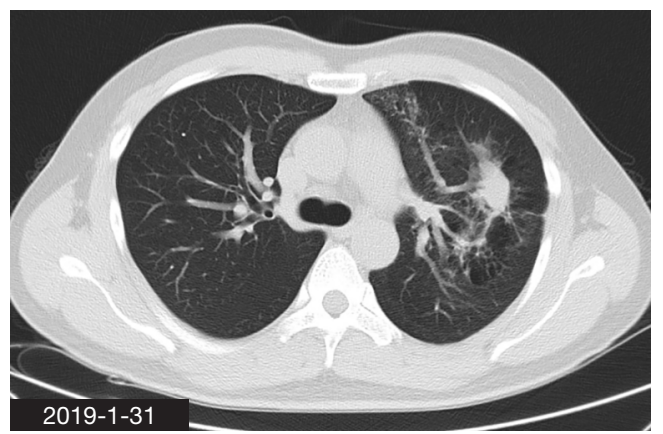

Figure 5 After 1 month treatment, the exudate in the left upper lobe was improved. The patchy mass in the left upper lobe was roughly the same as that on November 22, 2018, and the cavity disappeared.

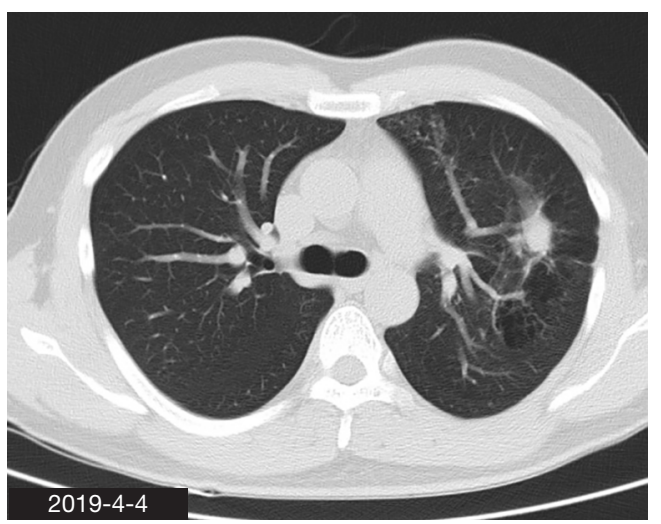

Figure 6 After 3 months treatment, the patchy mass in the left upper lung shrank remarkably.

lungs, and moist rales were heard in the left lung. Heart and abdomen examinations showed no abnormalities. No edema of lower extremities was observed.
Blood gas analysis showed $\mathrm{pH} 7.445, \mathrm{PaCO}_{2} 33 \mathrm{mmHg}$, $\mathrm{PaO}_{2} 106 \mathrm{mmHg}$, and $\mathrm{FiO}_{2}$ 21\%. White blood cells (WBC) count was $17.36 \times 10^{9} / \mathrm{L}$, percentage of neutrophils was $79.2 \%$; serum C-reactive protein was $39.94 \mathrm{mg} / \mathrm{L}$ (reference level: $<5 \mathrm{mg} / \mathrm{L}$ ), and the procalcitonin level was $0.05 \mathrm{ng} / \mathrm{mL}$. Tumor-associated carbohydrate antigen 19-9 was $27.43 \mathrm{U} / \mathrm{mL}$, and levels of cytokeratin, carcinoembryonic antigen, alpha-fetoprotein, and carbohydrate antigen 125 were normal. HIV antibody was negative. No growth of pathogenic bacteria was observed in two sputum cultures. Sputum smear microscopy for acid-fast organisms showed negative results. Blood tests for Cryptococcus and Aspergillus antigens, (1-3)- $\beta$-D-Glucan, and interferon-gamma release assay were negative, as well as antinuclear antibody, antidouble-stranded DNA antibody, and anti-neutrophil cytoplasmic antibody. Bronchoscopy showed tracheal and bronchial congestion. The acid-fast bacilli were negative in sputum smear harvested by bronchoscopy and by bronchial brush, and no abnormality was seen in the bronchial brush cytology. Bronchoalveolar fluid (BALF) sample was negative for tuberculosis (smear, PCR, and culture). BALF sent to Beijing Genomics Institution for high-throughput gene detection for pathogenic factors returned negative. Sputum culture under tracheoscopy revealed the presence of Actinomyces odontolyticus, which was 1+ during the semiquantitative assay, accounting for $90 \%$ of microorganisms.

After admission, the patient initially received moxifloxacin and cefoperazone sodium-sulbactam sodium. After the result of post-bronchoscopy sputum culture was obtained, the patient was switched to 4-million penicillin $\mathrm{q} 8 \mathrm{~h}$, and ornidazole $0.5 \mathrm{~g} \mathrm{q} 12 \mathrm{~h}$, both through intravenous infusion. Four weeks later, chest CT (Figure 5) revealed that, compared with previous CT, the exudate in left upper lobe was improved; although the patchy hypertense mass in the left upper lobe was roughly similar. Internal cavity had disappeared. The patient was discharged with oral amoxicillin $0.5 \mathrm{~g} \mathrm{q} 8 \mathrm{~h}+$ ornidazole $0.5 \mathrm{~g} \mathrm{q} 12 \mathrm{~h}$. After 9 weeks, the chest CT (Figure 6) revealed that, compared with previous CT, the patchy hypertense mass had decreased. After 6 months treatment (Figure 7), the patchy mass in the left upper lung get a further reduction.

\section{IMDT discussion}

\section{Discussion among physicians from Shenzhen People's Hospital, Shenzhen Institute of Respiratory Diseases}

Actinomycetes are Gram-positive anaerobic bacteria found 


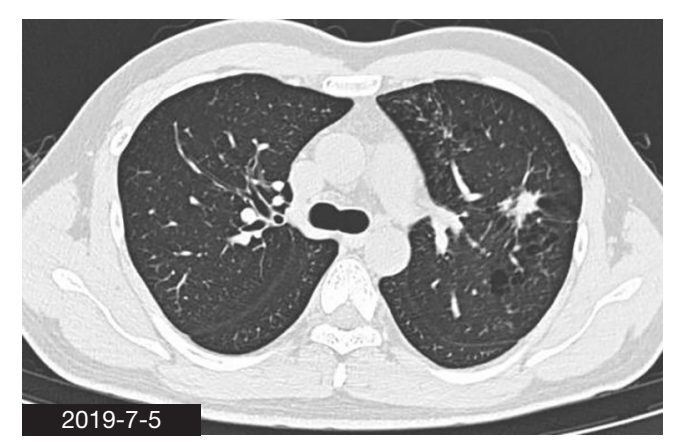

Figure 7 After 6 months treatment, the patchy mass in the left upper lung get a further reduction.

in the healthy mouth, dental caries, periodontal abscess, tonsillar crypts, gastrointestinal tract, and genitourinary tract. They are conditional pathogens (1). The clinical manifestations of PA are non-specific. The main symptoms include cough, sputum production, blood-stained sputum, and chest pain. Coughing up yellow granules, which are actinomycetes abscess formed by the entangling of actinomycetes, is a characteristic feature. The imaging findings of PA vary according to the different stages of the disease. In the early stage, it can manifest as bronchial pneumonia; in the later stages, the lesion can invade the pleura and chest wall, forming sinuses, which are often visualized as mass opacities. The typical chest CT finding is lung-limited gas-containing consolidation, which contains low-density areas with peripheral enhancement and adjacent pleural thickening (2-4). In rare cases, bronchial actinomycete infection can be accompanied by bronchiectasis, which is characterized by the worsening of localized bronchiectasis, irregular thickening of bronchial walls, and bronchial perivascular consolidation. There have also been reports of bronchial actinomycetes infections accompanied by bronchial stones or foreign bodies, which are classically observed as proximal calcified bronchial nodules or intrabronchial high-density nodules with distal obstructive consolidation (5).

As an aerobic or strictly anaerobic bacteria, actinomycetes need to be cultured in an anaerobic environment. Both symbiotic microorganisms and contamination with other microorganisms can inhibit the growth of actinomycetes, so the positive rate of sputum culture for actinomycetes is typically low (6). Moreover, if actinomycetes are found only in sputum, especially in the absence of sulfur granules, they may merely reflect colonization. Therefore, the diagnosis of actinomycosis is relatively tricky. A confirmed diagnosis is based on pathology. The identification of actinomycetes hyphae and sulfur granules in biopsy samples is the gold standard for diagnosis (1).

Due to the sclerosis of lesions, inadequate local blood supply, and poor penetration of drugs into local tissue, PA treatment often requires a long course. Penicillin (18 to 24 million units per day for 2-6 weeks) is the preferred treatment, followed by oral penicillin $\mathrm{V}$ or amoxicillin for 6-12 months. Other active drugs include doxycycline, chloramphenicol, erythromycin, azithromycin, clindamycin, ceftriaxone, and imipenem. Metronidazole, trimethoprimsulfamethoxazole, ceftazidime, oxacillin, are ineffective for actinomycetes (7). Surgical treatment is required in the presence of extensive tissue necrosis, fistula, sinus, unsuccessful antibiotic treatment, or if there is a need to remove malignant tumors.

The patient was a young man without any underlying disease. The medical condition has lasted for more than 2 years. The pulmonary lesions were found on chest CT examinations, and manifested as slowly-progressing cavities in upper lung. Following the onset of cough, progression of exudate in the lungs was observed. A chronic infectious disease, in particular tuberculosis, was suspected at admission. After repeated pathogen-related tests including smear and culture of sputum samples obtained by tracheoscopy, BALF, with additional testing for tuberculosis nucleic acid, and high-throughput gene detection of infectious pathogens, only Actinomyces odontolyticus was documented, by bronchoscopy, while all other tests yielded negative results. Since the disease progressed slowly in the case reported herein, a diagnosis of PA was finally made. The disease gradually improved after treatment with penicillin and ornidazole, supporting the diagnosis of PA.

In addition to the slowly-progressing cavitary lesions, chest imaging also showed local emphysema, pulmonary bullae, and abnormal bronchial vascular bundles in the left upper lung. In the early stage of the disease, gas retention following bronchial occlusion may lead to emphysema and pulmonary bulla, followed by an abnormal course of the bronchial vascular bundles. The cause of bronchial obstruction remains unclear. As mentioned earlier, bronchial actinomycete infection may occur with bronchial stones or foreign bodies, manifested as proximal bronchial calcification nodules or intrabronchial high-density nodules with distal obstructive consolidation (5). In the case reported herein, chest CT did not reveal bronchial calcification or foreign body, and ultra-thin bronchoscopy was not performed. Therefore, it can only be speculated 
that the disease may be related to actinomycete infection, but the evidence is not sufficient.

Several issues regarding the treatment of $\mathrm{PA}$ and this patient were further discussed as follows.

Question 1: With the advances in the detection methods, in particular, the wide application of molecular biology and bigh-throughput genetic detection technology, the diagnosis of PA may be obtained earlier. Should the treatment course of early PA (if manifested as bronchial pneumonia only) be shortened accordingly? Can the complete disappearance of the lesion under imaging be used as an indication for drug withdrawal?

\section{Expert opinion 1: Dr. Pierre Tattevin}

A 6-12 month-course of antibacterial treatment is the rule in extended PA, but the duration of treatment course may theoretically be shortened when PA is diagnosed earlier (e.g., when limited to bronchial pneumonia), or when large surgical resection was performed (8). Although the optimal duration of treatment has not been investigated through randomized trial, most experts would recommend to monitor the response to treatment, through follow-up imaging studies (e.g., every 3 months). It seems reasonable to prolong antibacterial treatment at least one month after the resolution of measurable active disease.

\section{Expert opinion 2: Dr. Goohyeon Hong}

Imaging of PA is not specific, and PA is frequently confused with malignancy (mass) or tuberculosis (cavitation). The golden standard for diagnosing PA is histopathological demonstration of sulphur granulesand bacterial culture of a lung biopsy, obtained by bronchoscopy, percutaneous biopsy guided by CT scan or by open surgical resection. Simple culture of Actinomyces in BAL, as with sputum, is inappropriate for the diagnosis of PA, except for patients with cavitation, as it may represent colonization. At early stages of the disease, a focal pulmonary consolidation occurs, which can be surrounded by pulmonary nodules, but there are often no associated physical symptoms at this stage. This primary pulmonary involvement could secondly lead to constitution of a peripheral mass, with or without cavitation, which could invade adjacent tissue. At this stage, PA is usually characterized by fibrotic lesion with slow contiguous growth passing through the anatomical barriers. The mass is often confused with malignancy. If PA was diagnosed in the early stage of the disease, there was no formation of fibrotic lesion with anatomical barriers. Therefore, no required prolonged high doses of antimicrobial therapy. However, case series involving patients with PA, treated exclusively medically with short course regimens, are sparse. Most patients with PA require prolonged high doses of antimicrobial therapy are frequently used because the accurate diagnosis is delayed. The duration of antimicrobials has to be individualized in patients with $\mathrm{PA}$, but that treatment durations less than 3 months in medically treated patients should be avoided, as these patients are at risk for recurrence or local complications. Termination of antibiotic treatment can probably be considered 1 or 2 months after complete clinical and radiological disease resolution in most patients.

\section{Question 2: PA is not susceptible to metronidazole.}

However, since actinomycetes are often present in mixed infections and metronidazole can kill the coexisting anaerobic bacteria, is it useful to include metronidazole in the combined therapy?

\section{Expert opinion 1: Dr. Pierre Tattevin}

Since actinomycetes are often present in mixed infections, it seems reasonable that initial antibacterial treatment includes drugs active on pathogens co-isolated with actinomycosis. Most commonly isolated co-pathogens include others strict anaerobes (Bacteroides fragilis, Fusobacterium spp., etc.), as well as HACEK bacteria (Haemophilus parainfluenzae, Aggregatibacter spp., Cardiobacterium spp., Eikenella corrodens, and Kingella spp.). In this observation, no other pathogen was identified, but it was decided to cover strict anaerobes that may be resistant to amoxicillin, by prolonged use of ornidazole. Although no robust data advocate for, or against, this empirical coverage of anaerobes, the duration of the combination could have been reduced, as co-pathogens don't require 6-12 months of treatment. In addition, cumulative toxicity of imidazoles may be severe and irreversible, especially peripheral neuropathy. Ornidazole could have been stopped after a three week-course, in my opinion.

\section{Expert opinion 2: Dr. Goohyeon Hong}

PA results mainly from aspiration of oropharyngeal or gastrointestinal secretions. Consequently, individuals with poor oral hygiene, preexisting dental disease, and alcoholism have an increased risk for developing PA. However, if there was no evidence of anaerobic bacterial infection such as positive culture from an adequate specimen, $i$ think that the combination therapy including metronidazole was not be 
required routinely in the concern of cost-effectiveness and increased antibiotic resistance.

Question 3: The patient loves sports and is very concerned about the protection of his lung function. Since local emphysema has occurred in the upper left lung, is it recommended to perform partial resection of the left upper lung to treat the lesions and preserve his lung function?

\section{Expert opinion 1: Dr. Pierre Tattevin}

Given the good response to antibacterial treatment, and the limited extension of lesions on the last CT scan, I would definitely not recommend surgery for this patient. High cure rates have been reported for PA managed with medical treatment alone. This patient has more chances to recover with good lung function without surgery, than with surgery.

\section{Expert opinion 2: Dr. Goohyeon Hong}

Lung volume reduction surgery (LVRS) aims to resect damaged emphysematous lung tissue, thereby increasing elastic properties of the lung. This treatment is hypothesised to improve long - term daily functioning and quality of life, although it may be costly and may be associated with risks of morbidity and mortality. LVRS is a surgical technique that may be beneficial for some patients with advanced emphysema who have poor control of their disease despite maximal medical therapy. Therefore, the patient's symptoms, exercise capacity and lung function are very important when deciding whether to have surgery. Result of pulmonary function test and dyspnea scale such as St George's Respiratory Questionnaire (SGRQ) is representative. LVRS may be an appropriate treatment for select patients who upper lobe-predominant emphysema and low exercise capacity. In this case, patient's lung lesion was located in the left upper lobe. Hence, surgery should be determined according to the results of his pulmonary function test and degree of dyspnea.

\section{Conclusions}

The diagnosis of PA relies on pathological examination, and the presence of actinomycetes hyphae and sulfur granules on biopsy can confirm the diagnosis. Only culture of Actinomyces in BAL, as with sputum, is insufficient for the diagnosis of PA, as it may represent colonization. At present, the underdiagnosed of PA is widespread partly due to the poor culture positive rate of anaerobes including actinomycetes. The preferred treatment is long-term, high-dose penicillin. Although a 6-12 month-course of antibacterial treatment is the rule in extended PA, the duration of antimicrobials should be individualized. The treatment durations less than 3 months in medically treated patients should be avoided. Termination of antibiotic treatment can probably be considered 1 or 2 months after complete clinical and radiological disease resolution in most patients.

\section{Acknowledgments}

None.

\section{Footnote}

Conflicts of Interest: The authors have no conflicts of interest to declare.

Ethical Statement: The authors are accountable for all aspects of the work in ensuring that questions related to the accuracy or integrity of any part of the work are appropriately investigated and resolved. Written informed consent was obtained from the patient for publication of this Case Report and any accompanying images.

\section{References}

1. Japanese Society of Chemotherapy Committee on guidelines for treatment of anaerobic infections; Japanese Association for Anaerobic Infection Research. Chapter 2-12-1. Anaerobic infections (individual fields): actinomycosis. J Infect Chemother 2011;17 Suppl 1:119-20.

2. Kim TS, Han J, Koh WJ, et al. Thoracic actinomycosis: CT features with histopathologic correlation. AJR Am J Roentgenol 2006;186:225-31.

3. Zhao W, Tian Y, Peng F, et al. Differential diagnosis of acute miliary pulmonary tuberculosis from widespreadmetastatic cancer for postoperative lung cancer patients: two cases. J Thorac Dis 2017;9:E115-20.

4. Heo SH, Shin SS, Kim JW, et al. Imaging of actinomycosis in various organs: a comprehensive review. Radiographics 2014;34:19-33.

5. Chouabe S, Perdu D, Deslee G, et al. Endobronchial actinomycosis associated with foreign body: four cases and a review of the literature. Chest 2002;121:2069-72.

6. Baron EJ, Miller JM, Weinstein MP, et al. Executive summary: a guide to utilization of the microbiology 
laboratory for diagnosis of infectious diseases: 2013 recommendations by the Infectious Diseases Society of America (IDSA) and the American Society for Microbiology (ASM)(a). Clin Infect Dis 2013;57:485-8.

7. Boyanova L, Kolarov R, Mateva L, et al. Actinomycosis: a frequently forgotten disease. Future Microbiol 2015;10:613-28.

8. Valour F, Senechal A, Dupieux C, et al. Actinomycosis: etiology, clinical features, diagnosis, treatment, and management. Infect Drug Resist 2014;7:183-97.

Cite this article as: Wang $\mathrm{L}$, Zhang $\mathrm{H}$, Wu D, Feng M, Yang P, Hu X, Tattevin P, Hong G, Chen R, Qiu C. Pulmonary lesions associated with sputum culture-positive actinomycetes: report of one case. Ann Transl Med 2019;7(23):793. doi: 10.21037/ atm.2019.12.38 